\title{
Non-Degenerate Four-Wave Mixing in a Silicon Nanowire and its Application for One- to-Six WDM Multicasting
}

Pu, Minhao; Hu, Hao; Ji, Hua; Galili, Michael; Oxenløwe, Leif Katsuo; Yvind, Kresten; Jeppesen, Palle; Hvam, Jørn Märcher

\section{Published in:}

Proceedings of the European Conference on Optical Communication (ECOC) 2011

Publication date:

2011

Document Version

Publisher's PDF, also known as Version of record

Link back to DTU Orbit

Citation (APA):

Pu, M., Hu, H., Ji, H., Galili, M., Oxenløwe, L. K., Yvind, K., Jeppesen, P., \& Hvam, J. M. (2011). NonDegenerate Four-Wave Mixing in a Silicon Nanowire and its Application for One-to-Six WDM Multicasting. In Proceedings of the European Conference on Optical Communication (ECOC) $2011 \mathrm{http}: / /$ www.ecoc2011.org/

\section{General rights}

Copyright and moral rights for the publications made accessible in the public portal are retained by the authors and/or other copyright owners and it is a condition of accessing publications that users recognise and abide by the legal requirements associated with these rights.

- Users may download and print one copy of any publication from the public portal for the purpose of private study or research.

- You may not further distribute the material or use it for any profit-making activity or commercial gain

- You may freely distribute the URL identifying the publication in the public portal 


\title{
Non-Degenerate Four-Wave Mixing in a Silicon Nanowire and its Application for One-to-Six WDM Multicasting
}

\author{
M. Pu, H. Hu, H. Ji, M. Galili, L. K. Oxenløwe, K. Yvind, P. Jeppesen, and J. M. Hvam \\ DTU Fotonik, Technical University of Denmark, Building 343, DK-2800 Lyngby, Denmark \\ mipu@fotonik.dtu.dk
}

\begin{abstract}
We present WDM multicasting based on non-degenerate four-wave mixing in a silicon nanowire. A one-to-six phase-preserving wavelength multicasting of $10 \mathrm{~Gb} / \mathrm{s}$ differential phaseshift-keying data is experimentally demonstrated with bit-error rate measurements.

OCIS codes: (190.4380) Nonlinear optics, four-wave mixing; (190.4390) Nonlinear optics, integrated optics; (060.4255) Networks, multicast; (130.7405) Wavelength conversion devices.
\end{abstract}

\section{Introduction}

Multicasting is an important feature in a communication network, which can efficiently send a stream of information from a single source to multiple destinations [1, 2]. By using multicasting technology, the amount of network resources can be significantly reduced compared to unicast only networks. Optical layer multicasting could be implemented at fixed wavelength by power splitting. An alternative is optical wavelength division multiplexing (WDM) multicasting, i.e., multicasting to different wavelengths. One of the most addressed techniques for WDM multicasting is all-optical multi-wavelength conversion (MWC), which replicates signals from one wavelength on multiple wavelengths. The WDM multicasting has been demonstrated in different devices including semiconductor optical amplifiers (SOAs) [3], electroabsorption modulators (EAMs) [4], and highly nonlinear fibers (HNLFs) [5, 6], based on different nonlinear effects such as self-phase modulation (SPM), cross-phase modulation (XPM), crossabsorption modulation (XAM), and four-wave-mixing (FWM).

Recently, wavelength conversion in silicon nanowires has attracted considerable research interest due to its compactness, large conversion bandwidth [7] and complementary metal-oxide-semiconductor (CMOS) compatibility. Silicon nanowires have been utilized to demonstrate wavelength conversion at data rates from 10 $\mathrm{Gb} / \mathrm{s}$ [8] to $640 \mathrm{~Gb} / \mathrm{s}$ [9], and a sixteen-way multicasting operation of $40 \mathrm{~Gb} / \mathrm{s}$ none-return-zero (NRZ) data has also been demonstrated in silicon nanowires [10]. However, sixteen continuous wave (CW) tunable laser sources (TLSs) are needed to act as input signals and phase information is not preserved in the converted signals. Very recently, a potential for multicasting operation based on non-degenerate FWM in a silicon nanowire has also been demonstrated [11]. However, in that demonstration only one-to-two wavelength conversion was achieved, and without bit-error rate (BER) validation.

In this paper, we report one-to-six phase-preserving WDM multicasting of $10 \mathrm{~Gb} / \mathrm{s}$ differential phase-shiftkeying (DPSK) data based on dual-pump non-degenerate FWM in a silicon nanowire. Error-free performances are achieved for all six multicast signals. Nine idlers are generated at the output of the silicon nanowire, which fully demonstrates the non-degenerate FWM in silicon. This is the first demonstration of WDM multicasting of a phase modulated signal in a silicon nanowire.

\section{Experiment}

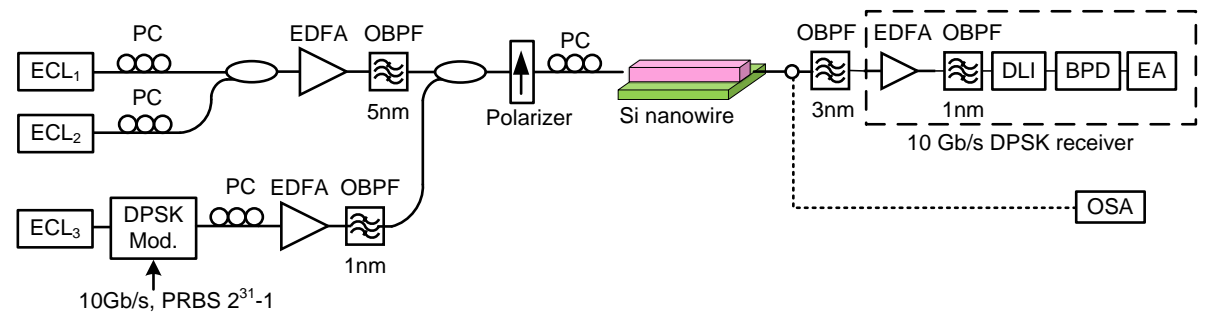

Fig. 1. Experimental setup for wavelength multicasting of $10 \mathrm{~Gb} / \mathrm{s}$ DPSK data in a silicon nanowire.

The experimental setup for the one-to-six WDM multicasting of a $10 \mathrm{~Gb} / \mathrm{s}$ DPSK signal in a silicon nanowire is shown in Fig. 1. Two pump waves are generated from two external cavity lasers (ECL ${ }_{1}$ and $\left.\mathrm{ECL}_{2}\right)$ at $1543.7 \mathrm{~nm}$ and $1547.5 \mathrm{~nm}$, respectively. They are combined by a 3-dB coupler, amplified by an erbium-doped fiber amplifier (EDFA), and then filtered by a 5-nm optical band pass filter (OBPF). The signal wave, generated by another external cavity laser $\left(\mathrm{ECL}_{3}\right)$ at $1533.5 \mathrm{~nm}$, is externally modulated by a Mach-Zehnder modulator with a $10 \mathrm{~Gb} / \mathrm{s}$ DPSK 
signal, encoded using a pseudo-random bit sequence (PRBS) of $2^{31}-1$. The signal wave is amplified by an EDFA, filtered by a 1-nm OBPF, and then combined with the two pump waves by a 3-dB coupler. Two tapered fibers are used for coupling light into and out from the silicon nanowire. The polarization controllers (PCs) and polarizer are used to align TE polarization into the silicon nanowire for the pump and signal waves. The silicon nanowire is 3-mm long and its cross-sectional dimension is $235 \times 450 \mathrm{~nm}^{2}$. It was fabricated on silicon-on-insulator (SOI) material using electron-beam lithography followed by reactive-ion etching. The silicon nanowire was inversely tapered at both ends and covered by a polymer waveguide for efficient coupling [12]. Following the silicon nanowire, one of the multicast signals is filtered out by a 3-nm OBPF and detected by the $10 \mathrm{~Gb} / \mathrm{s}$ DPSK receiver (shown by the dashed square in Fig. 1). In the receiver, the $10 \mathrm{~Gb} / \mathrm{s}$ DPSK data signal is pre-amplified, filtered with a 1-nm OBPF and then detected by a one-symbol delay interferometer (DLI). The output of the DLI is detected using a balanced photodetector (BPD), followed by a $10 \mathrm{~Gb} / \mathrm{s}$ error analyzer for BER measurement. An optical spectrum analyzer (OSA) is used to measure the output spectrum.

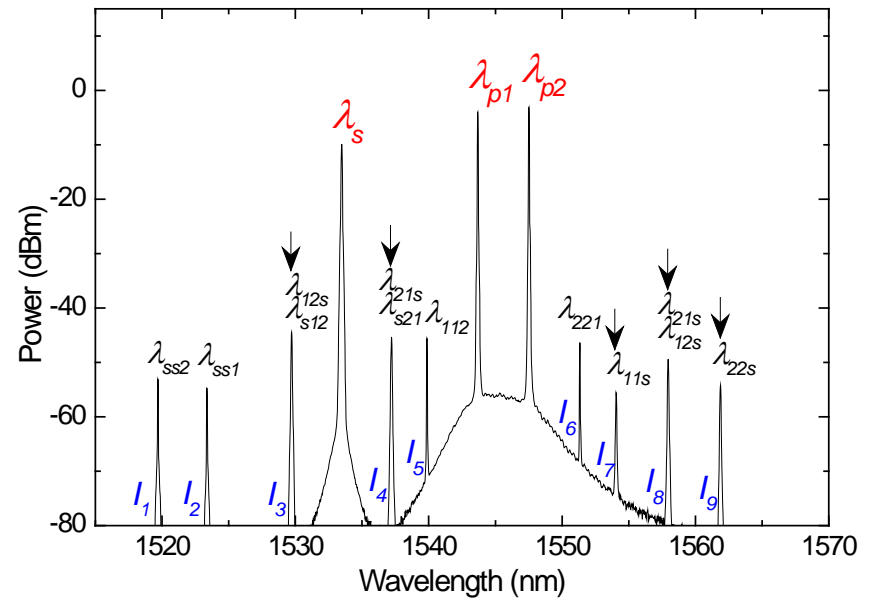

Fig. 2. Measured optical spectrum at the output of the silicon nanowire. The phase-preserved converted idlers are indicated by arrows.

Fig. 2 shows the spectrum measured at the output of the silicon nanowire. The two CW pump waves and the input signal wave are denoted $\lambda_{p 1}, \lambda_{p 2}$, and $\lambda_{s}$, respectively. The input powers of the two pumps and the signal are $13.2 \mathrm{~mW}, 16.2 \mathrm{~mW}$, and $9.5 \mathrm{~mW}$, respectively. Due to the dual-pump FWM process, nine idlers are generated as shown in Fig. 2. However, not all the converted idlers carry the same phase information as the original DPSK data. Here, we denote each FWM generated wave as $\lambda_{i j k}$, where the subscripts " $i$ " and " $j$ " refer to the two pump waves and " $k$ " refers to the signal wave in the corresponding FWM process. The phase information of the generated wave can be expressed as $\left(\Phi_{i}+\Phi_{j}-\Phi_{k}\right)$. To preserve the phase information of the original DPSK data in a generated FWM wave, there should be and can only be one wave that carries the original DPSK data $\left(\lambda_{s}\right)$ in the three wave components $\left(\lambda_{i}, \lambda_{j}, \lambda_{k}\right)$ of each FWM process. The generated nine idlers can be categorized into the non-degenerate $(i \neq j)$ and degenerate $(i=j)$ FWM-converted idlers. Each non-degenerate FWM-converted idler contains two overlapping non-degenerate FWM waves resulting in a higher FWM conversion efficiency compared with that of degenerate ones. All the three non-degenerate FWM converted idlers $\left(I_{3}, I_{4}\right.$, and $\left.I_{8}\right)$ satisfy the mentioned phase preserving requirement and thus can be decoded, resulting in the same phase information as the original DPSK data. However, not all the six degenerate FWM converted idlers $\left(I_{1}, I_{2}, I_{5}, I_{6}, I_{7}\right.$, and $\left.I_{9}\right)$ satisfy the phase preserving requirement. The phase information is not preserved for $I_{1}$ and $I_{2}$ since the original DPSK data signal wave $\left(\lambda_{s}\right)$ serves as the pump wave and acts twice in the FWM process. While there is no original DPSK data signal wave $\left(\lambda_{s}\right)$ involved in the FWM process for generating $I_{5}$ and $I_{6}$. Only two degenerate FWM converted idlers $I_{7}$ and $I_{9}$ carry the same phase information as the original DPSK data. Therefore, there are six output signals (including the output signal at $\lambda_{s}$ ) carrying the same phase information and a one-to-six multicasting of the DPSK signal is achieved.

To characterize the performance of the WDM multicasting, we measured the BER of the $10 \mathrm{~Gb} / \mathrm{s}$ back-to-back and the multicast $10 \mathrm{~Gb} / \mathrm{s}$ DPSK data signals, as shown in Fig. 3. Error-free operations were obtained for all the multicast signals. It is also seen that there is no error floor for all the converted idler signals. The maximum power penalty of the wavelength multicast signals is $3.8 \mathrm{~dB}$ at $10^{-9}$ for the converted idler $I_{7}$. The relatively large power penalty is mainly due to the residual CW pumps and low optical signal-to-noise ratio (OSNR) induced by the large amplified spontaneous emission (ASE) noise from the EDFA. In addition, the relatively low conversion efficiency for idler $I_{7}$ also contributes to the relatively large power penalty. The insets in Fig. 3 show the eye diagrams of the $10 \mathrm{~Gb} / \mathrm{s}$ DPSK data signals for the input signal (left) and the converted idler $I_{7}$ (right). It is seen that the converted 
idler signal also has clear and open eyes which are comfortably error free. The receiver sensitivity of the converted idler signals could be improved by better filtering away the residual CW pump or increasing the pump power which will result in an increased OSNR and increased conversion efficiency.

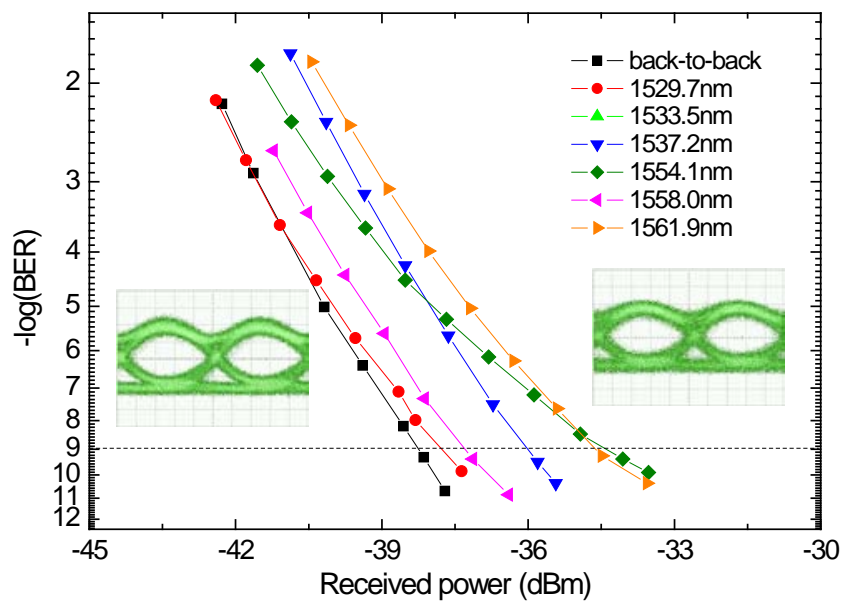

Fig. 3. BER measurement for the $10 \mathrm{~Gb} / \mathrm{s}$ back-to-back DPSK signal, and for the six multicast $10 \mathrm{~Gb} / \mathrm{s}$ DPSK signal. Insets show the eyediagrams of the input $10 \mathrm{~Gb} / \mathrm{s}$ data signal at $1533.5 \mathrm{~nm}$ (left) and the wavelength converted $10 \mathrm{~Gb} / \mathrm{s}$ data signal at $1554.1 \mathrm{~nm}$ (right).

Compared with wavelength multicasting based on degenerate FWM [10], the dual-pump non-degenerate FWM offers a more efficient technique since it reduces the number of required laser sources which simplifies the multicasting system and reduces the power consumption. In addition, the non-degenerate FWM-based multicasting in this work is phase-preserving and thus is suitable for multicasting of DPSK or even higher-level modulation formats. Since the DPSK data format has higher receiver sensitivity and a higher tolerance to fiber nonlinearities compared to the conventional on-off keying (OOK) data format [13], the phase-preserving non-degenerate FWM could be a promising wavelength multicasting technique for future WDM optical networks.

\section{Conclusion}

We have experimentally demonstrated all-optical one-to-six WDM multicasting of 10 Gb/s DPSK data in a 3-mm long silicon nanowire. The multicasting operation is based on non-degenerate FWM in silicon. The phase information of the input DPSK signal has been successfully preserved and delivered from one wavelength to six multicast wavelengths using two pumps. In the BER measurement, all the six multicast signals show error-free performance with a maximum power penalty of $3.8 \mathrm{~dB}$. The silicon nanowire could be useful for WDM multicasting applications in future WDM networks.

\section{Reference}

[1] X. Zhang, J. Wei, and C. Qiao, "Constrained multicast routing in WDM networks with sparse light splitting," J. Lightwave Technol., 18, Dec. 2000, pp. 1917-1927.

[2] R. K. Pankaj, "Wavlength requirements for multicasting in all-optical networks,” IEEE/ACM Trans. Networking 7, Jun. 1999, pp. 414-424.

[3] G. Contestabile, M. Presi, and E. Ciaramella, "Multiple wavelength conversion for WDM multicasting by FWM in an SOA," IEEE Photon. Technol. Lett., 16, Jul. 2004, pp. 1775-1777.

[4] L. Xu, N. Chi, K. Yvind, L. Christiansen, L. K. Oxenløwe, J. Mørk, P. Jeppesen, and J. Hanberg, "7×40 Gb/s base-rate RZ all-optical broadcasting utilizing an electroabsorption modulator,” Opt. Express, 12, pp. 416-420.

[5] G. Lu, K. S. Abedin, and T. Miyazaki, "DPSK multicast using multiple-pump FWM in Bismuths highly nonlinear fiber with high multicast efficiency,” Opt. Express, 16, Dec. 2008, pp. 21964-21970.

[6] C.S. Brès, A. O. J. Wiberg, B. P. P. Kuo, N. Alic, and S. Radic, "Wavelength Multicasting of 320-Gb/s Channel in Self-Seeded Parmmetric Amplifier," IEEE Photon. Technol. Lett., 21, Jul. 2009, pp. 1002-1005.

[7] A. C. Turner-Foster, M. A. Foster, R. Salem, A. L. Gaeta, and M. Lipson, "Frequency conversion over two-thirds of an octave in silicon nanowaveguides,” Opt. Express, 18, Feb. 2010, pp. 1904-1908.

[8] H. Rong, Y. H. Kuo, A. Liu and M. Paniccia, and O. cohen, "High efficiency wavelength conversion of $10 \mathrm{~Gb} / \mathrm{s}$ data in silicon waveguides," Opt. Express, 14, Feb. 2006, pp. 1182-1188.

[9] H. Hu, H. Ji, M. Galili, M. Pu H. C. H. Mulvad, L. K. Oxenløwe, K. Yvind, J. M. Hvam, and P. Jeppesen, ” Silicon Chip Based Wavelength Conversion of Ultra-High Repetition Rate Data Signals,” Proc. OFC 2011, PDPA8.

[10] A. Biberman, B. G. Lee, A. C. Turner-Foster, M. A. Foster, M. Lipson, A. L. Gaeta, and K. Bergman, "Wavelength multicasting in silicon photonic nanowires,” Opt. Express, 18, Aug. 2010, pp. 18047-18054.

[11] S. Gao, E. Tien, Q. Song, Y. Huang, and O. Boyraz, "Ultra-broadband one-to-two wavelength conversion using low-phase-mismatching four-wave mixing in silicon waveguides,” Opt. Express, 18, May. 2010, pp. 11898-11903.

[12] M. Pu, L. Liu, H. Ou, K. Yvind, and J. M. Hvam, "Ultra-low-loss inverted taper coupler for silicon-on-insulator ridge waveguide,” Opt. Commun., 283, Oct. 2010, pp. 3678-3682.

[13] A. H. Gnauck, and P. J. Winzer, “Optical phase-shift-keyed transmission,” J. Lightw. Technol., 23, Jan. 2005, pp. 115-130. 